\title{
Una revisión actualizada de las macroalgas marinas del Ecuador continental
}

\section{An updated review of marine macroalgae from continental Ecuador}

\section{Nancy Cuvi Fajardo ${ }^{*}$ \& Xavier Cornejo ${ }^{2}$}

\author{
${ }^{1}$ Consultora independiente \\ ${ }^{2}$ Departamento de Botánica, Facultad de Ciencias Naturales, Universidad de Guayaquil, Av. \\ Raúl Gómez Lince s.n. y Av. Juan Tanca Marengo (Campus Mapasingue), P.O. Box 09-01- \\ 10634, Guayaquil-Ecuador
}

Recibido de agosto 2020; recibido en forma revisada 26 de agosto 2020, aceptado 3 de octubre 2020 Disponible en línea 20 de diciembre 2020

\begin{abstract}
Resumen
Se realizó una revisión bibliográfica y de herbario para determinar el estado actual de las macroalgas marinas distribuidas en la zona intermareal a lo largo del perfil del Ecuador continental. En esta región se registran 4 filum: Rhodophyta (92 spp.), Chlorophyta (50 spp.), Ochrophyta (24 spp.) y Charophyta (1 sp.), éstos comprenden un total de 7 clases, 23 órdenes, 41 familias, 62 géneros y 167 especies distribuidas a lo largo de las 5 provincias costeras de Ecuador. Entre éstas, Florideophyceae (Rhodophyta) es la clase con mayor número de especies a nivel costerocontinental (90 de un total de $92 \mathrm{spp}$.), y la más diversa en cada una de las provincias analizadas. Charophyta con sólo una especie (Nitella, Characeae) se registra solamente en Esmeraldas. A nivel nacional continental, Esmeraldas es la provincia que registra mayor número de géneros (35) y especies (47). La costa del Ecuador continental comprende un área insuficientemente estudiada, el conocimiento ficológico en esta región se ha sextuplicado en los últimos 25 años, la generación de información taxonómica continúa en orden creciente.
\end{abstract}

Palabras claves: Ecuador continental, macroalgas, Pacífico.

\begin{abstract}
A bibliographic and herbarium review was carried out to determine the current status of marine macroalgae distributed in the intertidal zone along the profile of continental Ecuador. In this region 4 phyla are recorded: Rhodophyta (92 spp.), Chlorophyta (50 spp.), Ochrophyta (24 spp.) and Charophyta (1 sp.); these comprise a total of 7 classes, 23 orders, 41 families, 62 genera and 167 species distributed throughout the five coastal provinces of Ecuador. Among these, Florideophyceae (Rhodophyta) is the class with the highest number of species on coastal-continental level (90 out of a total of $92 \mathrm{spp}$. ), and the most diverse in each of the analyzed provinces. Charophyta with only one species (Nitella, Characeae) is only recorded in Esmeraldas. At the continental national level, Esmeraldas is the province with the highest number of genera (35) and species (47). The coast of continental Ecuador comprises an insufficiently studied area, the phycological knowledge in this region has increased sixfold in the last 25 years, the generation of taxonomic information continues in increasing order.
\end{abstract}

Keywords: Continental Ecuador, macroalgae, Pacific.

\section{Introducción}

Las algas son plantas marinas y dulceacuícolas no vasculares, constituyen componentes básicos de la producción primaria, ocupan el primer eslabón de la cadena alimentaria en el medio acuático, siendo las macroalgas las más grandes, estratificadas o filamentosas, que pueden llegar a formar bosques acuáticos. Estas brindan sustento y refugio a una amplia gama de especies desde invertebrados hasta vertebrados, creando espacios para comunidades complejas, interactuando además con el medio líquido y los sustratos. Las macroalgas son unos de los primeros agentes responsables de la producción de materia orgánica y energía, sobre éstas dependen los otros miembros de la cadena trófica. Como productores primarios, absorben nutrientes directamente del agua de mar para su crecimiento y desarrollo y utilizan

* Correspondencia del autor:

E-mail: nancy.cuvif@ug.edu.ec 
el dióxido de carbono disuelto y agua como materia prima en la producción de materia orgánica a través de los procesos de fotosíntesis (Müller \& Salazar, 1996).

Las macroalgas se encuentran distribuidas desde la zona intermareal hasta unos 100 metros de profundidad según sus requerimientos lumínicos, esto es, hasta donde penetran los rayos del sol; en esos ambientes colonizan y se desarrollan sobre diversos sustratos, para evitar ser arrastradas por las corrientes. Las algas bentónicas son parte importante de la producción primaria costera, entre éstas, las Phaeophyceae y Chlorophyta son los grupos más abundantes que se encuentran en aguas poco profundas, debido a que dependen de una mayor incidencia lumínica, este es uno de los factores más importantes para su crecimiento. Por otro lado, las Rhodophyta usualmente se encuentran más representadas a una mayor profundidad y presentan una menor abundancia (González \& Gabino, 2014). En éste último grupo, algunas especies calcáreas costrosas contribuyen como constructores de arrecifes y juegan un papel importante en la acumulación de carbono en las áreas marinas costeras, estas podrían ser utilizadas en la caracterización de la calidad del agua, convirtiéndose así en indicadores biológicos de contaminación y ciertas sustancias tóxicas (Müller \& Salazar, 1996).

Debido a las propiedades fitoquímicas, las macroalgas han sido objeto de investigación en las últimas décadas, siendo aprovechadas en alimentación y salud humana, como medicamentos, cosmetología, fertilizantes orgánicos y biocombustibles (Velasco, 2008).

Durante los pasados siglos diversos tipos de expediciones han sido realizadas con la finalidad de obtener información y recursos de la naturaleza. Inicialmente, los primeros naturalistas empezaron a documentar la historia natural obteniendo valiosas colecciones de rocas, animales y plantas, en distintas regiones del planeta. Desde finales del siglo XV hasta las primeras décadas del siglo XIX, parte considerable de la América tropical estuvo bajo la dominación española, durante este período, resaltan las investigaciones realizadas por el científico alemán Alexander von Humboldt, quien junto con el naturalista francés Aimé Bonpland y gracias al permiso y apoyo ofrecidos por el rey de España, emprendieron un extraordinario viaje al nuevo mundo (Ruales \& Cornejo, 2020; Sierra Praeli, 2019). Humboldt y Bonpland fueron pioneros en colectar macroalgas en la costa del Pacífico de Ecuador y mayormente de Perú, de las obtenidas en este último país, nueve especies fueron publicadas en el séptimo volumen de Nova Genera et Species Plantarum (1825: 418), sus colecciones comprenden los 3 tipos de novedades taxonómicas que fueron publicadas por Agardh (Howe, 1914).

Una las contribuciones más valiosas para la flora ficológica del Pacífico en América del Sur a inicios del siglo XX, es la realizada por Robert E. Cocker, experto en pesquerías del gobierno del Perú (Howe, 1914). Entre 1906 a 1908, Cocker realizó cientos de colecciones en la costa del Pacífico de Perú y envió los especímenes a Marshall Avery Howe, en aquel entonces curador del herbario NY en la ciudad de New York, obteniendo para la época una de las mayores colecciones de macroalgas de América del Sur. Estas fueron estudiadas y los resultados publicados en The marine algae of Perú (Howe, 1914), este trabajo contiene 28 nuevas especies, esta obra indirectamente contribuyó en cierto grado al conocimiento de la flora ficológica de Ecuador.

Posteriormente, una de las expediciones científicas más notorias es la del capitán George Allan Hancock en el Pacífico este a bordo del Velero III (1931-1941), las salidas comprendieron 775 estaciones realizadas durante las visitas a las costas de América central y noroccidente de América del Sur, incluyendo a las islas Galápagos como objetivo principal (Allan Hancock Foundation, 1940). Durante esta expedición se realizaron numerosas colecciones de macroalgas marinas, muchas de estas corresponden a nuevas especies descubiertas a lo largo de su trayectoria incluyendo las costas del Ecuador continental, éstas fueron principalmente descritas por William Randolph Taylor (Taylor, 1945), miembro de la expedición de Allan Hancock en el Pacífico occidental, quien diseñó la primera caracterización sobre la flora de algas marinas de las islas Galápagos. Taylor realizó caracterizaciones en diferentes puntos del continente, y reportó 35 especies de Chlorophyta, 34 especies de Phaeophyta y 137 especies de Rhodophyta; los especímenes tipo actualmente se encuentran en herbarios como UC/JEPS de California, NY, y US del Instituto Smithsoniano, entre otros (Taylor, 1945). Las publicaciones que surgieron producto de esta expedición permitieron elaborar listados de especies y posteriormente bases de datos en Latinoamérica, constituyendo una contribución al conocimiento de la composición de la flora marina del Pacífico este (op. cit.).

En la década de los 70's, Flor de María Valverde realizó una publicación denominada: “Vegetación marina de Ballenita, Península de Santa Elena Ecuador - Provincia del Guayas". En 1988, el biólogo australiano Gary Kendrick realizó una recopilación completa de las algas marinas bentónicas de las costas del Ecuador continental, en ese trabajo se describieron alrededor de 320 especies, indicando los endemismos y sus hábitats característicos. En 1994, el Instituto Nacional de Pesca (INP), realizó el proyecto de investigación denominado "Algas marinas del Ecuador”, en el recopilaron información sobre la vegetación marina del Ecuador continental y las islas Galápagos, y también reportó nuevas distribuciones de las macroalgas regionales.

Posteriores estudios ficológicos realizados a lo largo de la línea costera continental del Ecuador y en las islas Galápagos son: Algas marinas del Ecuador (Müller \& Salazar, 1996) y Macroalgas marinas (Garske, 2002). Entre trabajos no publicados, es necesario 
mencionar los esfuerzos realizados por la profesora (actualmente jubilada) MSc. Matilde Velasco (2013), con los estudiantes de la carrera de Biología de la Facultad de Ciencias Naturales de la Universidad de Guayaquil, quienes desde la década de los 80's han realizado importantes colecciones de macroalgas que reposan en la mencionada institución. Hasta la actualidad, varios tipos de trabajos o tesis realizadas por egresados de varias Universidades contemplan inventarios de macroalgas realizados principalmente en la zona intermareal de la costa del Ecuador continental (Rubira Carvache, 2012; Franco Campos, 2012; González \& Gabino, 2014; Pozo Rosales, 2014; Sánchez, 2020), sin embargo, la mayoría de estos no citan especímenes o colecciones de algas que reposen en herbario alguno como soporte de las taxa reportadas, consecuentemente, las identidades provistas en algunos casos no puede ser verificable.

En años recientes, el Departamento de Botánica de la Facultad de Ciencias Naturales de la Universidad de Guayaquil junto con estudiantes de botánica y otros colaboradores han realizado una de las colecciones más significativas de Corallinales costrosas (Rhodophyta) en la costa continental del Ecuador, los especímenes se encuentran en el Herbario GUAY y son objeto de estudio con microscopio electrónico de barrido y técnicas moleculares con colegas de varias instituciones en el extranjero, los resultados aún no están publicados.

Desde aproximadamente la década de 1970, el desarrollo de la biología molecular ha tenido injerencias con la investigación de la biología comparada, sin embargo, en los últimos 20 años, el uso de métodos moleculares y sus interpretaciones de aproximaciones han producido numerosos y significativos cambios en la sistemática, taxonomía y nomenclatura de algas en todas las jerarquías taxonómicas (Díaz Larrea, Pedroche \& Sentíes, 2016); sin embargo, en muchos casos la información producida se encuentra dispersa. Un ejemplo de actualizaciones de grupos taxonómicos con base en un número de investigaciones que han utilizado datos moleculares es el realizado en las Phaeophyceae (Silberfeld et al., 2014). En esta clase, la investigación en sistemática molecular en las últimas dos décadas no había logrado proporcionar a los ficólogos una clasificación clara, existiendo una acumulación de resultados sorprendentes y también en casos contradictorios, debido a la elevada plasticidad morfológica de las algas pardas, produciendo confusión y ambigüedades nomenclaturales.

Afortunadamente, en años recientes se ha propuesto una clarificada, organizada y definida clasificación que sirve como marco a nivel supragenérico (Silberfeld et al., 2014). Sin embargo, el esquema de clasificación en los restantes grupos de macroalgas aún se encuentra en construcción, siendo la base de datos algaebase (Guiry \& Guiry, 2021) un referente a nivel global.

Los nuevos cambios y novedades en las macroalgas marinas de la costa de Ecuador no se encuentran organizados y documentados en la literatura, siendo la información dispersa y desactualizada a nivel nacional. Con este antecedente, en este trabajo se presenta una revisión actualizada de la sistemática de las especies de macroalgas marinas del Ecuador continental, esta se encuentra organizada según los grupos taxonómicos vigentes a luz de los datos moleculares, morfológicos, citológicos y de ciclos de vida más recientes.

\section{Materiales y Métodos}

\section{Área de estudio}

El área de estudio comprende la línea costera continental del Pacífico ecuatoriano que tiene una extensión de aproximadamente $1.200 \mathrm{~km}$ de longitud, desde el río Mataje al norte hasta el río Zarumilla al sur (Varela \& Ron, 2018), con énfasis en la zona intermareal. Este perfil costero abarca las provincias de: Esmeraldas, Manabí, Santa Elena, Guayas y El Oro (fig. 1).

Los recursos que se pueden utilizar para recolectar y extraer información acerca de las macroalgas marinas ecuatorianas incluyen colecciones del Herbario GUAY de la Universidad de Guayaquil y se realizaron consultas on line de Algaebase www.algaebase.org (Guiry \& Guiry, 2021) y de especímenes del Herbario NY del Jardín Botánico de Nueva York. Se revisaron los registros disponibles de trabajos como Algas Marinas del Ecuador (Müller \& Salazar, 1996), Algas bentónicas del Ecuador Península de Santa Elena (Velasco, 2013) y diferentes investigaciones y tesis con bases y registros de macroalgas de la región disponibles online.

\section{Referencias históricas}

La historia de la investigación botánica en el Ecuador continental ha sido brevemente descrita (Müller \& Salazar, 1996). Entre los trabajos más relevantes se puede mencionar a la contribución de la expedición de Allan Hancock en su visita a las costas de Ecuador (Taylor, 1945), hay diversas publicaciones producto de las colecciones realizadas. Se incluyeron investigaciones de diferentes autores, como Boedeker et al. (2016, 2017), Lam \& Zechman (2006), etc.

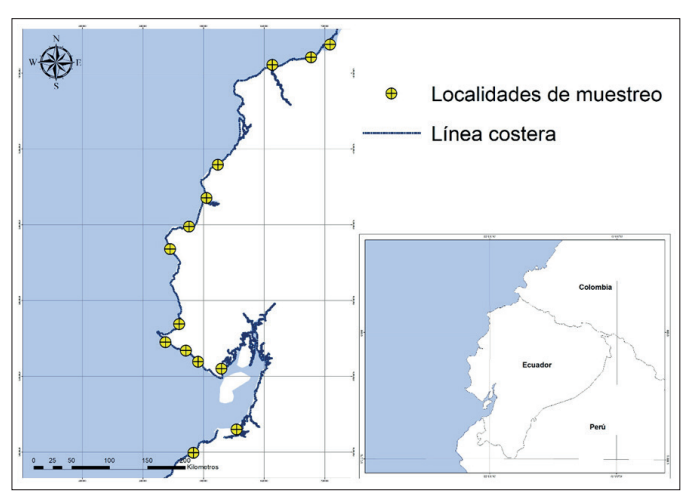

Figura 1. Perfil costero del Ecuador continental. Cortesía de Anthony Albán. 


\section{Lista de especies}

Para elaborar una lista comprensiva de las especies presentadas en este trabajo se revisó la colección de macroalgas marinas del herbario GUAY y se realizó la revisión de las taxa presentadas en Algas Marinas Del Ecuador (Müller \& Salazar, 1996), este libro comprende las especies presentes en las áreas costeras del Ecuador continental y en algunas zonas costeras de las islas Galápagos, presenta una larga lista que necesita ser revisada y actualizada. También se revisaron publicaciones y tesis (Garske, 2002; Abbott \& Hollenberg, 1976; Velasco, 2013; Cárdenas-Calle et al., 2020; Sánchez, 2020; RincónDíaz, et al., 2021; Bajaña, 2021; Vera, 2021), cuyos resultados han contribuido en la elaboración de la lista base presentada en este trabajo. Los cambios y actualizaciones taxonómicas aquí presentadas han sido consultados, confirmados o comparados con Algaebase www.algaebase.org (Guiry \& Guiry, 2021).

\section{Sistematización de la información}

Toda la información que se presentó en este trabajo fue agrupada en una plantilla para macroalgas del Ecuador continental, diseñada en Microsoft Excel. Esta tiene como objetivo principal brindar una herramienta accesible, dinámica y precisa. Se presentan los nombres actualizados de las taxa y a partir de estos se obtuvieron cuadros comparativos de especies, órdenes, clases y phylum a nivel continental y provincial.

\section{Colecciones de herbario}

Se revisaron los especímenes depositados en el Herbario GUAY donde reposan las colecciones realizadas por la MSc. Matilde Velasco con sus estudiantes de la Facultad de Ciencias Naturales desde la década de los 80s; otras colecciones de macroalgas son las realizadas en años recientes por Xavier Cornejo con sus estudiantes de Botánica I y algunos colaboradores. Algunos especímenes de algas calcáreas del género Lithophyllum (Corallinales, Rhodophyta) han sido identificados por imágenes SEM de microscopio electrónico de barrido y molecularmente por Joe Richards (University of Lousiana at Lafayette).

\section{Análisis estadístico}

Por último, se aplicó un índice de similitud BrayCurtis, agrupando las provincias en relación a las clases registradas dentro de ellas, bajo la siguiente fórmula:

$$
\begin{aligned}
& \text { n } \\
& \Sigma|x i j-x j k| \\
& \mathrm{Djk}=\frac{i=1}{\sum(x i j+x i k)} \\
& i=1
\end{aligned}
$$

Donde:

Djk = valor de similitud entre las unidades de muestreo.

$\mathrm{n}$ = número de individuos en la especie

Xij y Xik = abundancia de la especie $i$ en la unidad de muestreo j y k respectivamente.

\section{Resultados}

La costa del Pacífico del Ecuador continental alberga 4 filos: Rhodophyta (92 spp.), Chlorophyta (50 spp.), Ochrophyta (24 spp.) y Charophyta (1 sp.; fig. 16-18), éstos comprenden un total de 7 clases, 23 órdenes, 41 familias, 62 géneros y 167 especies de macroalgas marinas distribuidas en la zona intermareal a lo largo del perfil de las 5 provincias costeras de Ecuador (fig. 2). Entre éstas, Florideophyceae perteneciente al filo Rhodophyta es la clase con mayor número de especies en todas las provincias analizadas. Se presenta un cuadro comparativo de las taxa a nivel provincial (fig. 3). El listado comentado de las especies de macroalgas marinas se encuentra en Cuvi (2021: anexos 1-5, disponible en: http://repositorio.ug.edu. ec/bitstream/redug/52770/1/Cuvi_Nancy_Tesis.pdf).

Un total de 23 órdenes de macroalgas marinas se registran en las costas del Ecuador continental, estos se encuentran distribuidos en todas las provincias costeras (figs. 2, 4).

\section{Similitud de Bray Curtis}

Aplicando el índice de Bray Curtis al número de especies registradas en cada provincia costera del Ecuador continental se aprecian dos subclados, uno que comprende a Esmeraldas, Manabí y Santa Elena, donde se concentra la mayor diversidad ficológica marina a nivel continental, y otro que manifiesta similitud entre Guayas y El Oro, que son las provincias con menor número de especies registradas (fig. 5).

Revisando los patrones de distribución de las macroalgas marinas en la costa del Pacífico este, hay un número que presentan sus rangos de distribución hasta cerca de los límites norte o sur del Ecuador continental. Considerando que las colecciones de macroalgas aún son insuficientes para documentar toda la flora marina continental y ante la gran posibilidad de incrementar los registros con futuras investigaciones y programas de colección, éstas han sido aquí incluidas (fig. 6; anexo 1), debido a que eventualmente podrían ser registradas en este país.

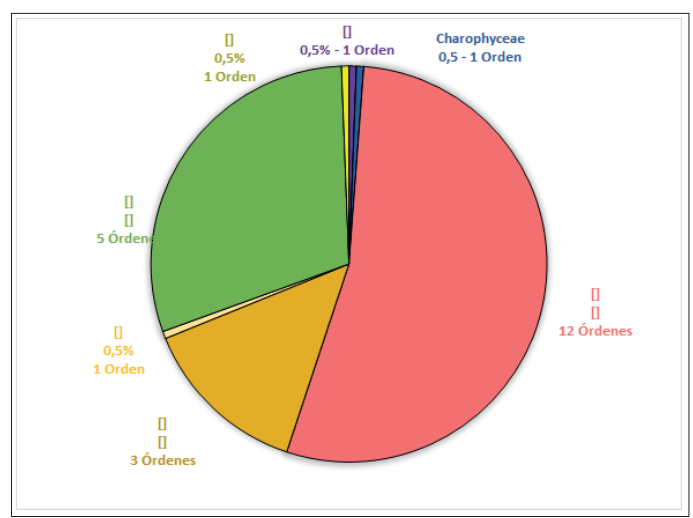

Figura 2. Representación en porcentajes de las clases, órdenes y especies de macroalgas marinas distribuidas en la costa del Ecuador continental. 


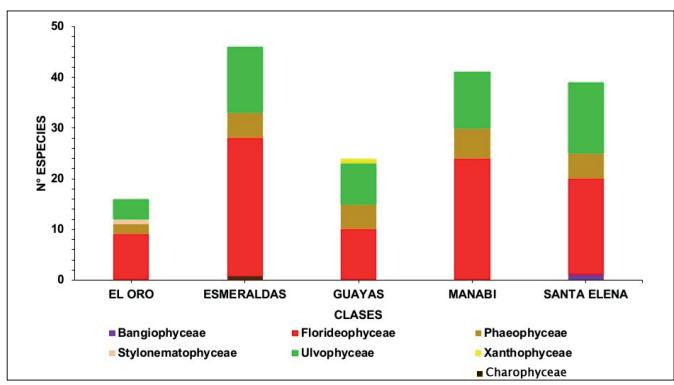

Figura 3. Número de especies de macroalgas marinas a nivel de clase registradas en las provincias costeras del Ecuador continental.

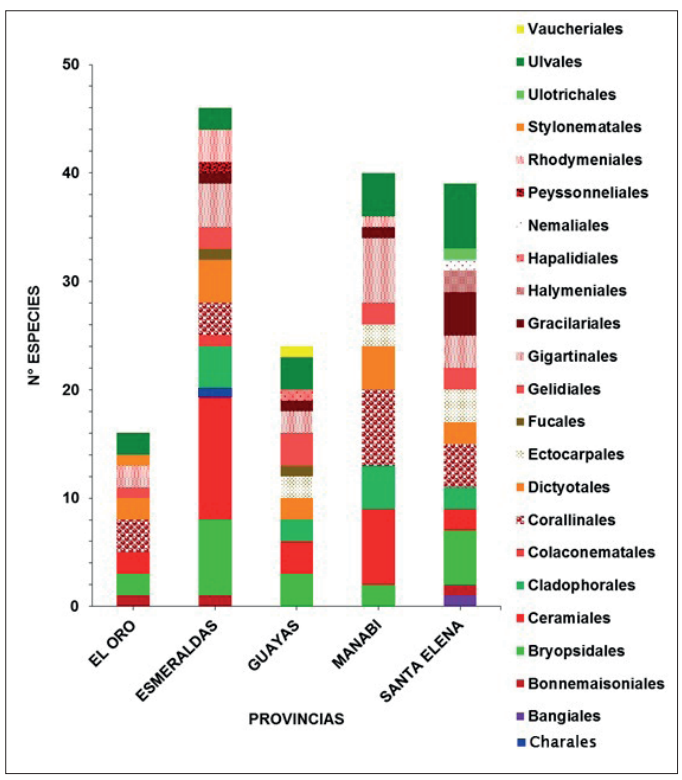

Figura 4. Número de especies de macroalgas marinas a nivel de orden registradas en las provincias costeras del Ecuador continental.

La provincia de Esmeraldas está representada por 4 clases, 15 órdenes, 29 familias, 35 géneros y 47 especies de macroalgas marinas. Florideophyceae perteneciente al phyllum de las Rhodophyta es la clase más diversa, ésta registra 9 órdenes, 18 familias, 21 géneros y 28 especies (fig. 7 , anexo 2 ). Charophyta con sólo una especie de Nitella (Characeae, Charales, Charophyceae), es el único filo registrado solamente en Esmeraldas (Cornejo \& Cangá 8281, GUAY, NY). Entre las contribuciones más recientes para esta provincia están las de Rincón-Díaz et al.et al. (2021) y Vera (2021). Según los especímenes que reposan en herbarios, desde la perspectiva ficológica esta es la provincia menos explorada del Ecuador continental. En un futuro cercano se esperan nuevos registros y aún nuevas especies. La provincia de Manabí cuenta con 3 clases, 12 órdenes, 18 familias, 27 géneros y 41 especies de macroalgas marinas. Florideophyceae perteneciente a las Rhodophyta es la clase más diversa, ésta se encuentra representada por 7 órdenes, 11 familias, 17 géneros y 24 especies (fig. 8 ,

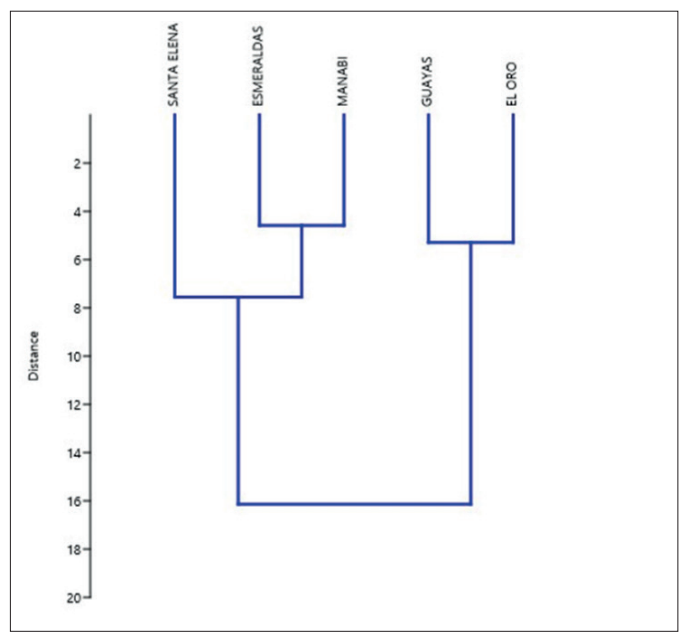

Figura 5. Similitud del número de especies de macroalgas marinas que presentan las provincias del Ecuador continental según Bray-Curtis.

anexo 3). Entre las contribuciones más recientes para esta provincia están las de Sánchez (2020) y Bajaña (2021).

La provincia del Guayas es la segunda con menos especies reportadas, ésta cuenta con 4 clases, 11 órdenes, 17 familias, 18 géneros y 24 especies de macroalgas marinas. Florideophyceae perteneciente al phyllum de las Rhodophyta es la clase más diversa, ésta se encuentra representada por 5 órdenes, 8 familias, 8 géneros y 10 especies (fig. 9, anexo 4). Ulvaceae (Chlorophyta) es la familia más diversa con 1 género y 3 especies.

La provincia de Santa Elena es la más diversa a nivel de géneros registrados, ésta cuenta con 4 clases, 15 órdenes, 24 familias, 29 géneros y 39 especies de macroalgas marinas. Posee una diversidad considerable de Rhodophyta y Chlorophyta. Las Rhodophyta se encuentran representada por 9 órdenes, 14 familias, 17 géneros y 20 especies; las Chlorophyta se encuentran representadas por 4 órdenes, 7 familias, 7 géneros y 14 especies (fig. 10, anexo 5). Según los especímenes que reposan en herbarios (Velasco, 2013), esta es la provincia más colectada del Ecuador continental.

La provincia de El Oro es la que menor diversidad a nivel de géneros registra, ésta cuenta con 4 clases, 11 órdenes, 13 familias, 15 géneros y 16 especies de macroalgas marinas. Posee una diversidad considerable de Rhodophyta y Chlorophyta. Las Rhodophyta se encuentran representadas por 8 órdenes, 9 familias, 10 géneros y 10 especies; las Chlorophyta se encuentran representadas por 2 órdenes, 3 familias, 3 géneros y 4 especies (fig. 11, anexo 6).

La clase Florideophyceae es la más representativa por estar compuesta de un mayor número de especies 
Rev. Cient. Cien. Nat. Ambien. 14(2):201-209 Diciembre 2020
Cuvi \& Cornejo • Una revisión actualizada de las macroalgas marinas del Ecuador continental

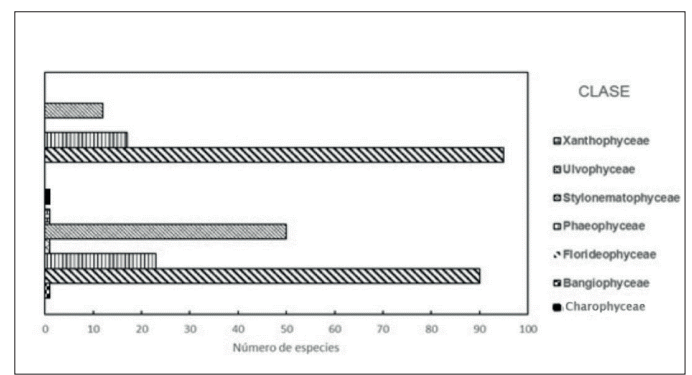

Figura 6. Potenciales nuevos registros a nivel de clase de las macroalgas marinas en la costa continental del Ecuador.

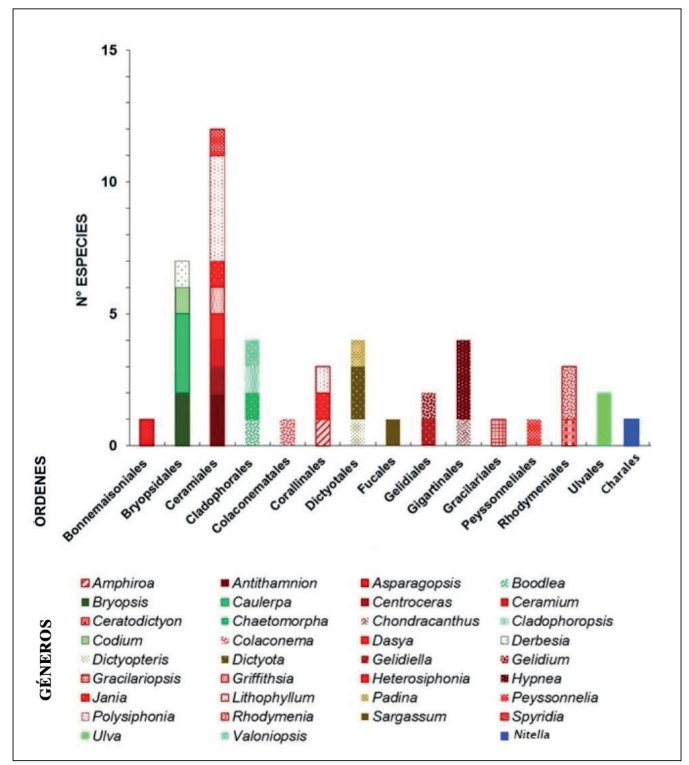

Figura 7. Órdenes, géneros y número de especies de macroalgas marinas registradas en la provincia de Esmeraldas.

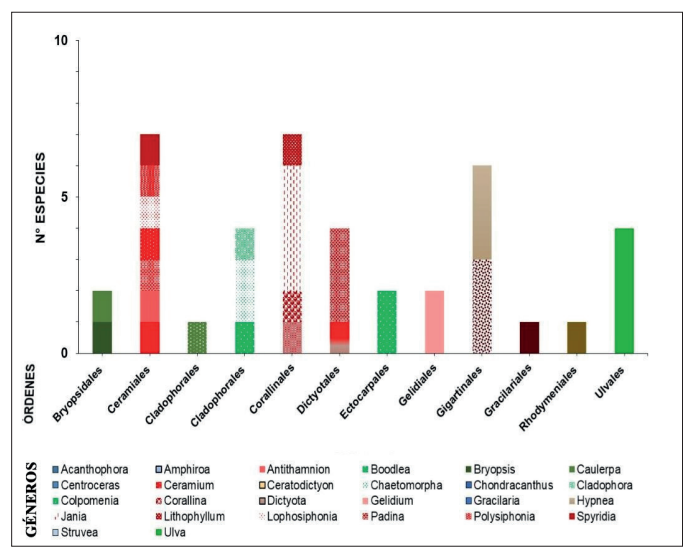

Figura 8. Órdenes, géneros y número de especies de macroalgas marinas registradas en la provincia de Manabí.

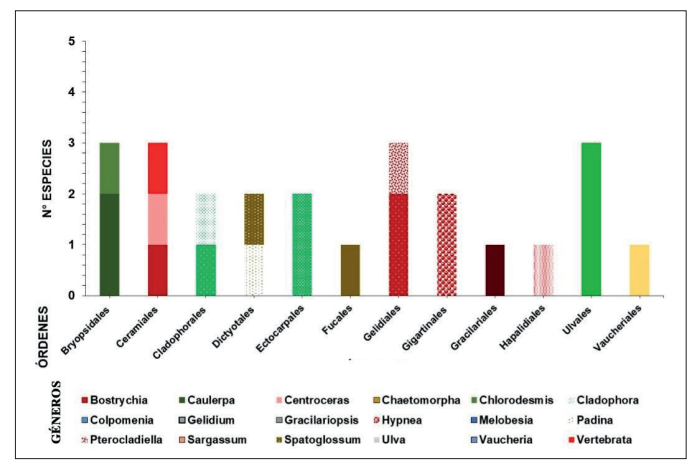

Figura 9. Órdenes, géneros y número de especies de macroalgas marinas registradas en la provincia del Guayas.

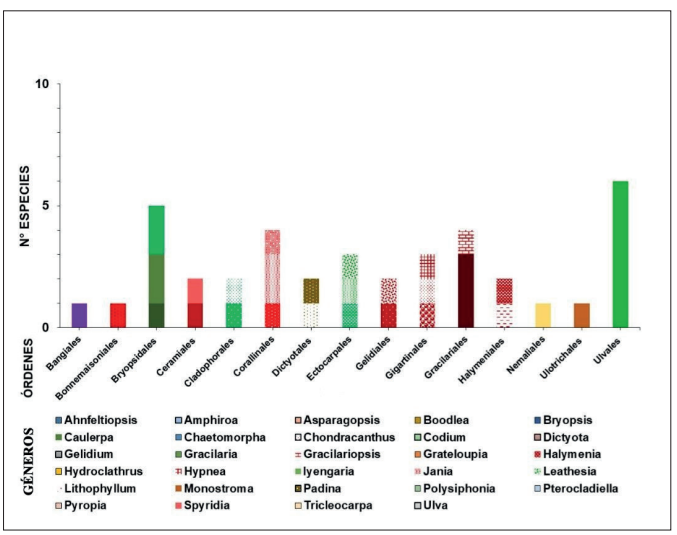

Figura 10. Órdenes, géneros y número de especies de macroalgas marinas registradas en la provincia de Santa Elena.

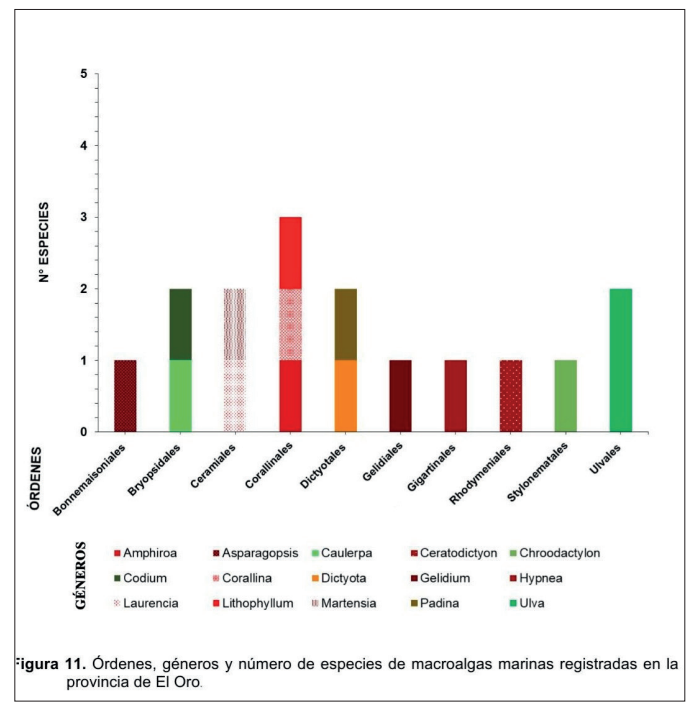

Figura 11. Órdenes, géneros y número de especies de macroalgas marinas registradas en la provincia de El Oro. 


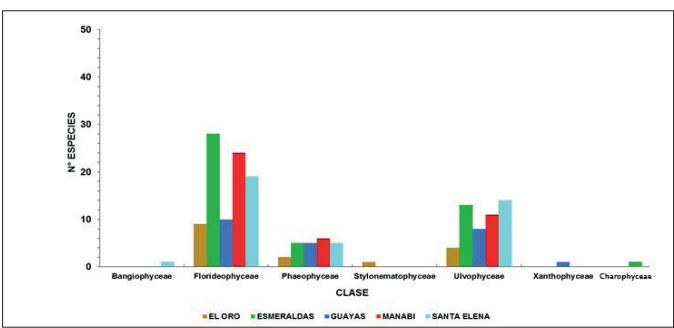

Figura 12. Número de especies según las clases de macroalgas marinas en las provincias costeras del Ecuador continental.

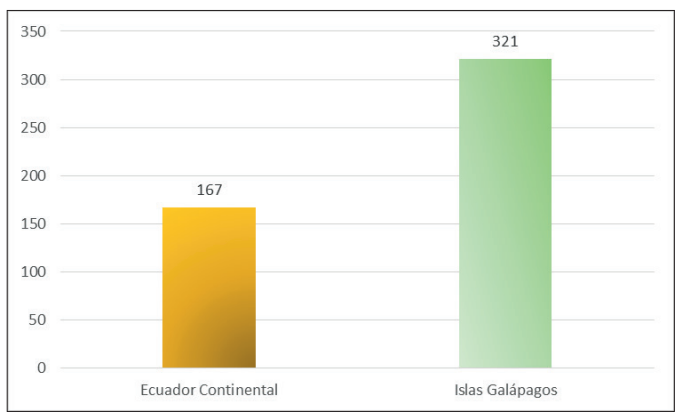

Figura 13. Diferencia del número de especies de macroalgas marinas entre la costa del Ecuador continental y las Islas Galápagos.

en todas las provincias de la costa continental del Ecuador. A nivel provincial, las Florideophyceae presentan la mayor diversidad en la provincia de Esmeraldas, esta es seguida por Manabí (figs. 5, 12). Las clases Ulvophyceae y Phaeophyceae se encuentran presentes con menor número de especies en todas las provincias. Las clases restantes, Xanthophyceae (Guayas), Bangiophyceae (Santa Elena) y Stylonematophyceae (El Oro), cada una se encuentra presente en una provincia de manera específica (fig. 12).

La provincia de Santa Elena presenta la mayor diversidad de clases, en orden decreciente ésta es seguida por Manabí, Guayas, Esmeraldas y El Oro (fig. 12).

En la presente investigación se pudo determinar que la diversidad de macroalgas marinas de la costa continental del Ecuador (167 spp.) es inferior en comparación con las Islas Galápagos (321 spp.; fig. 13).

Al comparar los resultados obtenidos en cuanto al número y composición de las taxa de macroalgas marinas en las provincias de la costa del Ecuador continental (Esmeraldas 46 spp., Manabí 41 spp., Santa Elena 39 spp., Guayas 24 spp. y El Oro 16 spp.), es evidente que esta es excedida notoriamente por la diversidad de la provincia de Galápagos (321 spp.; Avila \& Padilla, 2020; fig. 14).

En el presente trabajo se registran 167 especies de macroalgas marinas en la costa del Ecuador continental. Las investigaciones de la flora ficológica en el Ecuador continental presentan resultados que

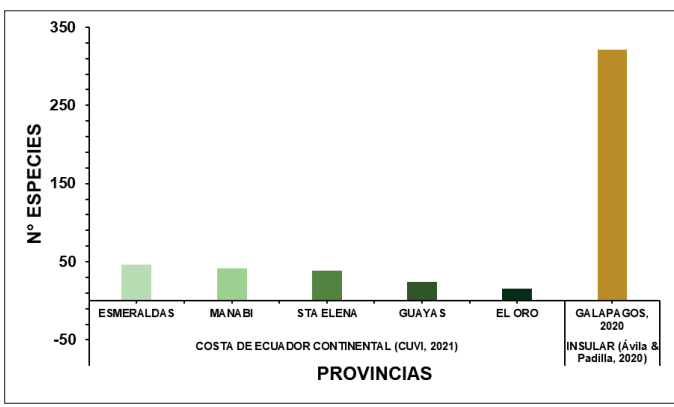

Figura 14. Comparación de la diversidad de macroalgas marinas en las provincias del Ecuador continental e insular (Islas Galápagos).

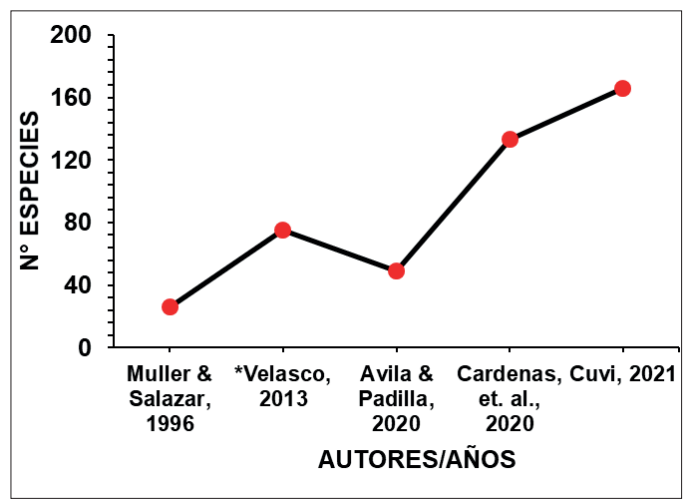

Figura 15. Avance en el conocimiento de la diversidad de macroalgas marinas del Ecuador continental (*Velasco 2013, es un trabajo no publicado que solo trata de la Península de Santa Elena).

han ido en ascenso gradual, habiéndose sextuplicado el número de taxa reportadas durante los últimos 25 años (fig. 15).

\section{Discusión}

La presente revisión y actualización sistemática de las macroalgas en la costa continental del Pacífico de Ecuador refleja los avances producidos en la diversidad, nomenclatura, taxonomía y fitogeografía en esta región. Trabajos realizados décadas atrás en el campo de la ficología poseen valores históricos, sin embargo, la capacidad de información confiable que podrían proveer actualmente es bastante limitada y en ocasiones hasta confusa al ser comparados con los resultados presentes. Esto se debe a la falta de colecciones ficológicas y al menor número de investigaciones que existían en este campo décadas atrás, existiendo numerosos cambios y aportes producidos como nuevos registros y descubrimiento de nuevas especies en la flora marina en esta región del Pacífico este en años recientes. Para citar ejemplos, en el libro Algas Marinas del Ecuador (Müller y Salazar, 1996), se reportó 26 especies para la costa continental del Ecuador, incluyendo sólo una especie en Manabí (versus 40 spp. en este trabajo) y una en Esmeraldas (versus 48 spp. en este trabajo); 
recientemente 49 especies (Avila-Peltroche y J. Padilla-Vallejos, 2020) son reportadas para la costa continental de Ecuador, estos resultados contrastan significativamente con las 167 especies de macroalgas aquí registradas. Además, al comparar en sentido cronológico las publicaciones de las macroalgas en Ecuador es evidente que la investigación ficológica a nivel nacional e internacional ha estado mayormente dirigida a las Islas Galápagos (op. cit.), mientras que en la costa continental el conocimiento ficológico es aún limitado (Avila-Peltroche y J. PadillaVallejos, 2020) y al parecer aún se encuentra en una etapa más temprana de desarrollo y en una era de descubrimientos aún creciente (fig. 15).

La elevada diversidad y predominancia de Florideophyceae (Rhodophyta) en la costa del Pacífico del Ecuador continental concuerda con la riqueza biológica de esta clase a nivel global (Yang et al., 2016). En grupos taxonómicos como las Corallinales costrosas (Rhodophyta) estudios morfoanatómicos son insuficientes y análisis moleculares e imágenes SEM de microscopio electrónico de barrido son fundamentales para la identificación a nivel de especie (Richards et al., 2017), estas técnicas frecuentemente son limitantes en la investigación a nivel nacional. Por otro lado, en la revisión de Caulerpa asistida con técnicas moleculares (Caulerpaceae, Chlorophyta; FernandezGarcía et al., 2016), se reportan 6 especies para la región del Pacífico Tropical Este, entre estas solamente $C$. racemosa (Forrskål) J. Agardh para las costas de Ecuador continental. Sin embargo, en el presente trabajo se registran 3 especies de Caulerpa en las costas de Ecuador continental ( $C$. racemosa, C. chemnitzia (Esper) J.V. Lamouroux y C. serrulata (Forsskål) J. Agardh), estas especies han sido frecuentemente colectadas desde los $80 \mathrm{~s}$ por estudiantes de la Facultad de Ciencias Naturales y numerosos especímenes de estas taxa reportadas reposan en el Herbario GUAY de la Universidad de Guayaquil. En este caso, es evidente que las limitaciones en consultas de herbarios regionales y locales pueden producir sesgos y ausencia de información. Es indudable que las colecciones de herbario y el rol que desempeñan las Universidades en el campo de la investigación han producido y producen los mayores aportes al conocimiento ficológico en Ecuador.

\section{Conclusiones}

La diversidad de las macroalgas marinas en la costa del Ecuador continental está representada por 4 phylum: Rhodophyta (92 spp.), Chlorophyta (50 spp.), Ochrophyta (24 spp.) y Charophyta (1 sp.), éstos comprenden un total de 7 clases, 23 órdenes, 41 familias, 62 géneros y 167 especies. En cuanto a número de especies por ecosistema este resultado podría compararse a la diversidad total de plantas vasculares registrada en los manglares en la misma región (179 spp.; Cornejo, 2014).
La diversidad ficológica marina en la costa del Ecuador continental (167 spp.) es superada en proporción 1,9: 1 por la del Ecuador insular presente en las Islas Galápagos (321 spp.; Avila-Peltroche y J. PadillaVallejos, 2020). La mayor diversidad de las Galápagos está explicada por el elevado nivel de endemismo producto de procesos evolutivos que se intensifican en islas, sin embargo, también hay que considerar que las investigaciones taxonómicas de la flora marina no se han producido con la misma intensidad en la costa de Ecuador y que su conocimiento aún está en una fase más temprana de construcción en esta región (fig. 13 y 14).

\section{Recomendaciones}

- Realizar revisiones taxonómicas a nivel de familias y géneros de las especies de macroalgas presentes en la línea costera del Ecuador continental, las especies reportadas o citadas deben estar respaldadas por especímenes que reposan en herbarios.

- Estudios moleculares de las macroalgas son necesarios para dilucidar los linajes genéticos y contribuir a definir a las entidades taxonómicas a nivel de especie y también en jerarquías supraespecíficas.

- Realizar colecciones de macroalgas marinas en las provincias menos estudiadas como Esmeraldas y El Oro, con la finalidad de tener un mejor nivel de conocimiento de la real riqueza ficológica.

- Evaluar el estado de las poblaciones de las macroalgas marinas del Ecuador continental, con énfasis en aquellas especies que poseen rangos angostos de distribución. La categorización de las especies en peligro es una prioridad como una herramienta que contribuya a su manejo y conservación.

- Realizar estudios fitoquímicos de las macroalgas marinas del Ecuador continental para determinar sus componentes, usos potenciales y aplicaciones en campos como medicina, alimentación e industria.

\section{Bibliografía}

Abbott, I. A., y Hollenberg, G. J. 1976. Marine Algae of California. California: Stanford University Press, Stanford, $828 \mathrm{pp}$, Vol 1.

Allan Hancock Foundation. 1940. Allan Hancock Pacific Expeditions. Vol. 2, 1935-1940. Retrieved from http:// archive.org/details/allanhancockpaci02alla

Avila-Peltroche, J. y J. Padilla-Vallejos. 2020. The seaweed resources of Peru. Botánica Marina, 63: 381-394.

Bajaña, C. 2021. Variación estacional en la composición de macroalgas en la zona mesolitoral rocosa de San Mateo, Manabí-Ecuador. Trabajo de titulación. Facultad de Ciencias Naturales, Universidad de Guayaquil.

Boedeker, C., Leliaert, F., y Zuccarello, G. C. 2016. Molecular phylogeny of the Cladophoraceae (Cladophorales, Ulvophyceae), with the resurrection of Acrocladus Nägeli 
and Willeella Børgesen, and the description of Lubrica gen. nov. and Pseudorhizoclonium gen. nov. Belgium: $\mathrm{H}$. Verbruggen (Associate Editor).

Boedeker, C., Leliaert, F., y Zuccarello, G. 2017. Genetic diversity and biogeography in Chaetomorpha melagonium (Ulvophyceae, Cladophorales) based on internal transcribed spacer (ITS rDNA) sequences. Botanica Marina, 60: 319-325.

Cárdenas-Calle, M., Mora, E., Torres, G., Pérez-Correa, J., Bigatti, G., Signorelli, J., y Coronel, J. 2020. Marine invertebrate and seaweed biodiversity of continental coastal Ecuador. Biodiversity Data Journal, 8: 1-52.

Coelho, F. 2020. Significados. Retrieved from https://www. significados.com/sinonimo/

Cornejo, X. 2014. Plants of the South American Pacific Mangrove Swamps (Colombia, Ecuador, Peru). Ed. EduQuil. Universidad de Guayaquil. Ecuador. 310 p.

Cuvi, N. 2021. Revisión actualizada de la sistemática de las macroalgas del Ecuador continental. Trabajo de titulación previo a la obtención del grado de Bióloga. Facultad de Ciencias Naturales, Universidad de Guayaquil. http:// repositorio.ug.edu.ec/handle/redug/52770

Díaz Larrea, J., Pedroche, F., y Sentíes, A. 2016. Impacto de los estudios moleculares en la ficología comparada mexicana: el caso de las macroalgas marinas. Revista de investigación y difusión sobre algas Cymbella, 2: 1-24.

Fernandez-Garcia, C., Wysor, B., Riosmena-Rodriguez, R., Pena-Salamanca, E. y Verbruggen, H. 2016. DNA-assisted identification of Caulerpa (Caulerpaceae, Chlorophyta) reduces species richness estimates for the Eastern Tropical Pacific. Phytotaxa, 252(3): 185-204.

Franco Campos, A. D. 2012. Caracterización ecológica de las comunidades intermareales rocosas a lo largo de una gradiente de productividad en las costas del Ecuador. Repositorio Institucional de la Pontificia Universidad Católica del Ecuador, 1era Edicion, 1-75.

Fredericq, S., Hommersand, M. H., y Freshwater, D. W. 1996. The molecular systematics of some agar-and carrageenan-containing marine red algae based on $\mathrm{rbcL}$ sequence analysis. En Fifteenth International Seaweed Symposium (pp. 125-135). Springer, Dordrecht.

Garske, L. E. 2002. Macroalgas marinas. En: Danulat, E. y Edgar, G.J. (eds.)(2002): Reserva Marina de Galápagos. Línea Base de la Biodiversidad, 419-439.

González, S. y Gabino, S. 2014. Diversidad y abundancia de macroalgas en las zonas intermareales rocosas de las comunas Montañita, La Entrada y La Rinconada, de noviembre 2013, abril 2014, provincia de Santa Elena. Repositorio Institucional Universidad Estatal Península de Santa Elena, 1-236.

Guiry, M. D. y G. M. Guiry. 2021. AlgaeBase. World-wide electronic publication National University of Ireland, Galway. Retrieved from https: / /www.algaebase.org

Howe, M. A. 1914. The marine algae of Peru. Memoirs of the Torrey Botanical Club, 15: 1-185.

Lam, D. W., y Zechman, F. W. 2006. Phylogenetic analyses of the Bryopsidales (Ulvophyceae, Chlorophyta) based on rubisco large subunit gene sequences. Phycological Society of America, 42: 669-678.

Martínez Centelles, V. 2019. Botanical-online. Retrieved from https://www.botanical-online.com/botanica/ nomenclatura-botanica-normas

Miladi, R., ManGhisi, A., MInICAntec, S., Genovesea, G., Abdelkafi, S., y Morabito, M. 2018. A DNA barcoding survey of Ulva (Chlorophyta) in Tunisia and Italy reveals the presence of the overlooked alien U. ohnoi. Cryptogamie, Algologie, 85-107.

Müller, H., y Salazar, M. 1996. Algas marinas del ecuador. Guayaquil: Comisión Asesora Ambiental - Instituto Nacional de Pesca.

Phillips, N., Burrowes, R., Rousseau, F., De Reviers, B., y Saunders, G. W. 2008. Resolving evolutionary relationships among the brown algae using chloroplast and nuclear genes 1. Journal of Phycology, 44(2): 394-405.

Pozo Rosales, M. 2014. Biodiversidad de macroalgas en los bajos “52, casa lobos y aquapark", de la Remacopse demostrando la importancia y dominancia de géneros a diferentes profundidades, durante los meses de agosto 2013 a enero 2014. Repositorio Institucional Universidad Estatal Peninsula de Santa Elena, 1-131.

Richards, J.L., Sauvage, T., Schmidt, W.E., Fredericq, S., Hughey, J.R. y Gabrielson, P.W. 2017. The coralline genera Sporolithon and Heydrichia (Sporolithales, Rhodophyta) clarified by sequencing type material of their generitypes and other species. Journal of Phycology, 53(5): 1044-1059.

Rincón-Díaz, N., Muñoz, J. V. S., Gavio, B., y Chasqui, L. 2021. Diversity of benthic macroalgae in the Colombian Pacific: a study of a rocky reef flora. Nova Hedwigia, 1-15.

Ruales, C. y Cornejo, X. 2020. La expedición de Humboldt y Bonpland en la antigua provincia de Guayaquil en Ecuador. Ed. Abya-Yala. Quito, Ecuador, 237 pp.

Rubira Carvache, K. 2012. Diversidad, abundancia y distribución de las macroalgas en la zona intermareal rocosa en las playas de Salinas, La Libertad y Ballenita (Península de Santa Elena-Ecuador, Octubre-Noviembre 2009). Repositorio Institucional de la Universidad de Guayaquil, 1era Ed., 1-93.

Sánchez Bermeo, C. 2020. Variación espacio-temporal en la composición de macroalgas en la zona intermareal rocosa en Salango, provincia de Manabí, Ecuador. Repositorio Institucional de la Universidad de Guayaquil, 1era Ed., 1-53.

Sierra Praeli, Y. 2019. Mongabay. Retrieved from https:// es.mongabay.com/2019/05/alexander-von-humboldtlegado/

Sigwart, J.D. 2018. What Species Mean: A User's Guide to the Units of Biodiversity. CRC Press, Vol 1, 232 páginas.

Silberfeld, T., Leigh, J. W., Verbruggen, H., Cruaud, C., De Reviers, B., y Rousseau, F. 2010. A multi-locus timecalibrated phylogeny of the brown algae (Heterokonta, Ochrophyta, Phaeophyceae): Investigating the evolutionary nature of the "brown algal crown radiation". Molecular Phylogenetics and Evolution, 56(2): 659-674.

Silberfeld, T., Rousseau, F., y de Reviers, B. 2014. An updated classification of brown algae (Ochrophyta, Phaeophyceae). Cryptogamie, Algologie, 35(2): 117-156.

Taylor, W. R. 1945. Pacific Marine Algae of the Allan Hancock Expeditions to the Galapagos Islands (Plates 1-100). Los Angeles: University of Southern California Press.

TURLAND, N. J., J. H. WIERSEMA, F. R. BARRIE, W. GREUTER, D. L. HAKSWORTH, P. S. HERENDEEN, S. KNAPP, W. H. KUSBER, D. Z. LI, K. MARHOLD, Y T. W. MAY. 2018. International Code of Nomenclature for Algae, Fungi, and Plants (Shenzhen Code). Adopted by the Nineteenth International Botanical Congress, Shenzhen, China, July 2017. Koeltz Botanical Books, Königstein.

Varela, L. A. y Ron, S. R. 2018. Geografía y clima del Ecuador. BIOWEB. Pontificia Universidad Católica del Ecuador.

Velasco, M. 2008. Introducción al estudio de las algas. Universidad de Guayaquil. 1er Ed., 213 pp.

Velasco, M. 2013. Algas bentónicas del Ecuador Península de Santa Elena, Catálogo de Géneros más representativos de Algas en la Península de Santa Elena. Universidad de Guayaquil. Guayaquil, Ecuador, 26 pp.

Vera, N. 2021. Composición y estructura de macroalgas en la zona mesolitoral rocosa de Súa, provincia de Esmeraldas, Ecuador. Trabajo de titulación. Facultad de Ciencias Naturales, Universidad de Guayaquil.

Yang, E. C., Boo, S. M., Bhattacharya, D., Saunders, G. W., Knoll, A. H., Fredericq, S., Graf, L. y Yoon, H.S. 2016. Divergence time estimates and the evolution of major lineages in the florideophyte red algae. Scientific Reports, 6(1): 1-11 